\title{
Analysis of KRAS Mutations of Exon 2 Codons 12 and 13 by SNaPshot Analysis in Comparison to Common DNA Sequencing
}

\author{
Rica Zinsky, ${ }^{1}$ Servet Bölükbas, ${ }^{2}$ Holger Bartsch, ${ }^{1}$ Joachim Schirren, ${ }^{2}$ \\ and Annette Fisseler-Eckhoff ${ }^{1}$
${ }^{1}$ Institute of Pathology and Cytology, HSK Wiesbaden, Academic Hospital of University of Mainz, Ludwig-Erhard-Strasse 100, 65199 Wiesbaden, Germany
${ }^{2}$ Department of Thoracic Surgery, HSK Wiesbaden, Academic Hospital of University of Mainz, Ludwig-Erhard-Strasse 100, 65199 Wiesbaden, Germany

Correspondence should be addressed to Rica Zinsky, zinsky@pathologie-wiesbaden.de

Received 24 September 2010; Revised 10 November 2010; Accepted 10 November 2010

Academic Editor: Antoni Castells

Copyright () 2010 Rica Zinsky et al. This is an open access article distributed under the Creative Commons Attribution License, which permits unrestricted use, distribution, and reproduction in any medium, provided the original work is properly cited.

\begin{abstract}
Due to the call for fast KRAS mutation status analysis for treatment of patients with monoclonal antibodies for metastatic colorectal cancer, sensitive, economic, and easily feasible methods are required. Under this aspect, the sensitivity and specificity of the SNaPshot analysis in comparison to the commonly used DNA sequencing was checked. We examined KRAS mutations in exon 2 codons 12 and 13 with DNA sequencing and SNaPshot analysis in 100 formalin-fixed paraffin-embedded tumor tissue samples of pancreatic carcinoma, colorectal carcinoma, and nonsmall cell lung cancer specimens of the primary tumor or metastases. $40 \%$ of these samples demonstrated mutated KRAS genes using sequencing and SNaPshot-analysis; additional five samples (45/100) were identified only with the SNaPshot. KRAS mutation detection is feasible with the reliable SNaPshot analysis method. The more frequent mutation detection by the SNaPshot analysis shows that this method has a high probability of accuracy in the detection of KRAS mutations compared to sequencing.
\end{abstract}

\section{Introduction}

Colorectal cancer, nonsmall cell lung cancer (NSCLC), and pancreatic carcinomas are three of the most frequent causes of cancer mortality in the world.

Established therapies are targeted on the epidermal growth factor receptor (EGFR), such as Panitumumab [1-4] and Cetuximab [5-8] (two monoclonal antibodies) treatment for metastatic colorectal carcinoma, Gefitinib for NSCLC, or Erlotinib for NSCLC and for pancreatic carcinoma. Treatment success using Cetuximab and Panitumumab for treatment of metastatic colorectal cancer depends on a nonmutated KRAS gene; the treatment is ineffective if the KRAS gene has any mutations $[1,5,6,8]$, which leads to an activated G-protein even in absence of the ligand, like the epidermal growth factor $[9,10]$. This results in further malignant proliferation of the cells despite treatment.
Two codons in the KRAS gene are mainly known to generate alternated proteins that are constitutively activated without the signal of a ligand bound to the EGFR. This are the codons 12 and 13 in the exon 2 of the KRAS gene $[9,10]$. Both codons encode the amino acid glycine in the wild type protein. Replacement of one of the first two bases leads in both codons to an amino acid exchange in the KRAS protein, resulting in resistance of the tumor to the above-described treatment.

Replacement of glycine leads to resistance of the GAPs, which are proteins causing the hydrolysis of KRAS bound GTP to GDP. The inability of GAPs to effect the GTP hydrolysis in mutated KRAS leads to a constitutive active protein [10].

Up to now KRAS mutation analysis is mainly performed by DNA sequencing [5] and commercialised test systems by DNA-DNA hybridisation (e.g., Chipron, Berlin, Germany), 
pyrosequencing [11], or the Therascreen: K-RAS Mutation Kit from Qiagen [12] based on Real-time PCR technologies. In consideration of an inhomogeneous genotype concerning the KRAS mutation status of the tumor cells, more sensitive detection methods are required. If the tumor has a small content of KRAS mutated cells, not detectable by DNA sequencing, anti-EGFR therapy probably fail to have an antiproliferating effect on these cells.

The SNaPshot analysis is regarded as more reliable in comparison to common DNA sequencing [8] and able to detect tiny allele amounts of mutated KRAS. The aim of this study is to test the SNaPshot method in comparison to DNA sequencing for screening of KRAS mutation.

\section{Materials and Methods}

2.1. Tumor Tissue Samples and DNA Extraction. Our study was carried out using tumor tissue samples from different sources: normal diagnostic cases of NSCLC $(n=41,33$ surgically resected specimens and 8 biopsy specimens) and colorectal carcinoma $(n=20,18$ surgically resected specimens and 2 biopsy specimens), archived pancreatic carcinomas ( $n=19,8$ surgically resected specimens and 11 biopsy specimens), and FFPE (formalin-fixed paraffinembedded) material of colorectal cancer specimens on slides from an interlaboratory comparison of KRAS mutation screening in spring 2008, organised by the German Society of Pathology. All specimens were made anonymous and examined by a pathologist, who choose a tumor-area with at least $70 \%$ of vital tumor cells for DNA-isolation. Weichert et al. [13] mentioned that specimens habroring fewer than $10 \%$ tumor cells showed lower mutation rates regardless of the method used.

The tumor tissue was fixed on slides ( $3 \mu \mathrm{m}$ thick), deparaffinated, and the tissue material was microdissected for DNA extraction using the QIAamp DNA Micro Kit (Qiagen, Hilden, Germany). Tissue material from the interlaboratory comparison on slides was identified by microscopy as tumor and normal tissue and DNA was extracted from both tissue samples $(n=20)$.

2.2. Amplification Step before SNaPshot and Sequencing Analysis. After extraction of the genomic DNA from the samples, the KRAS gene exon 2 was amplified by PCR (with HotStarTaq DNA Polymerase, Qiagen, Hilden, Germany) with the following primer set: $5^{\prime}$-AAGGCCTGCTGAAAATGACTG-3' and 5'-CAAAGAATGGTCCTGCACCAG-3' [8]. After checking the amplification product on an agarose gel, the PCR reaction was purified with the MinElute PCR Purification Kit (Qiagen, Hilden, Germany) and used for sequencing and the $\mathrm{SNaPshot}$ reaction.

Each PCR reaction had a $25 \mu \mathrm{L}$ volume and contained: the above-mentioned primer set with a concentration of $400 \mathrm{nM}, 1 \times$ PCR buffer, $2.5 \mathrm{mM} \mathrm{MgCl}_{2}, 0.1 \mu \mathrm{g} / \mu \mathrm{L}$ BSA, 5 units of the polymerase, $200 \mu \mathrm{M}$ of each deoxynucleotide, and $100 \mathrm{ng}$ of genomic DNA. The PCR reaction ran with the following program: $95^{\circ} \mathrm{C}$ initial denaturation for activating the HotStarTaq DNA Polymerase for 15 minutes, step 2 denaturation at $94^{\circ} \mathrm{C}$ for 30 seconds, step 3 annealing of the primer to the template at $55^{\circ} \mathrm{C}$ for 30 seconds, and step 4 30 seconds at $72^{\circ} \mathrm{C}$ for primer extension. Steps 2 to 4 were repeated 45 times followed by a final extension step at $72^{\circ} \mathrm{C}$ for 10 minutes, then the PCR reaction was cooled down to $8^{\circ} \mathrm{C}$. One PCR preparation is used for sequencing reaction and the SNaPshot analysis.

2.3. DNA Sequencing. The purified DNA was used as a template for the sequencing reaction with the BigDye Terminatorv1.1 Cycle Sequencing Kit (Applied Biosystems, Forster City, CA). The sequencing reaction was performed with the $3^{\prime} k R A S$ primer (5'-CAAAGAATGGTCCTGCACCAG-3') with the following program on the thermocycler: 1 minute for an initial denaturation of the DNA at $96^{\circ} \mathrm{C}$, followed by 25 cycles of a 10 -second denaturation at $96^{\circ} \mathrm{C}$, annealing of the primer at $50^{\circ} \mathrm{C}$ for 5 seconds, and the extension step at $60^{\circ} \mathrm{C}$ for 4 minutes. After this program, the samples were stored at $4^{\circ} \mathrm{C}$.

The capillary electrophoresis was performed on the 3130 Genetic Analyzer, and the obtained electropherogram (Figure 1(b)) was analysed with finchTV (Geospiza, Seattle, WA, USA) software (receivable online).

2.4. SNaPshot. The primers used for SNaPshot were implemented accordingly to the algorithm of Di Fiore et al. [8]: codon 12 position 1; 5'-AACTTGTGGTAGTTGGAGCT3', codon 12 position 2; 5'-GATCGTACTTGTGGTAGTTGGAGCTG-3', codon 13 position 1; 5'-GATCGATCGATCTTGTGGTAGTTGGAGCTGGT-3', codon 13 position 2; 5'-GATCGATCGATCGATCGATGTGGTAGTTGGAGCTGGTG-3'. These primers anneal one nucleotide position before the potential mutated base on the template DNA and in the course of polymerization with the SNaPshot Multiplex Kit (Applied Biosystems, Forster City, CA, USA), only this nucleotide is added as di-deoxy nucleotide to the primer sequence. A further polymerization is excluded, because of the lacking $3^{\prime}$-hydroxyl group on their nucleotide deoxyribose.

The SNaPshot reaction took place in a $10 \mu \mathrm{L}$ volume with $1 \mu \mathrm{L}$ PCR product, $5 \mu \mathrm{L}$ SNaPshot Multiplex Master Mix, and a $2 \mu \mathrm{M}$ concentration of each primer. The program on the thermocycler was 25 cycles of denaturation at $96^{\circ} \mathrm{C}$ for 10 seconds, primer-annealing at $50^{\circ} \mathrm{C}$ for 5 seconds, and an extension step at $60^{\circ} \mathrm{C}$ for 30 seconds then cooling down to $4^{\circ} \mathrm{C}$. After the SNaPshot reaction was performed, a purification step was necessary to eliminate interferingfree nucleotides. Adding $1 \mu \mathrm{L}$ shrimp alkaline phosphatase $(1 \mathrm{U} / \mu \mathrm{L})(\mathrm{NEB}$, Ipswich, MA) and incubating for 1 hour at $37^{\circ} \mathrm{C}$, followed by a denaturation step at $75^{\circ} \mathrm{C}$ for $15 \mathrm{~min}$, clean up the $\mathrm{SNaPshot} \mathrm{reaction} \mathrm{for} \mathrm{capillary} \mathrm{electrophoresis}$ on the 3130 Genetic Analyzer (Applied Biosystems, Forster City, CA).

For capillary electrophoresis an internal DNA sizing ladder was used, the GeneScan-LIZ 120 Size Standard (Applied Biosystems, Forster City, CA). No further analysis program is necessary; the relevant data can be read directly from the electropherogram [8] (e.g., Figure 1(a)). 


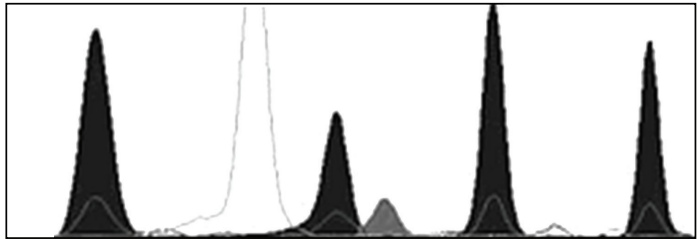

(a)

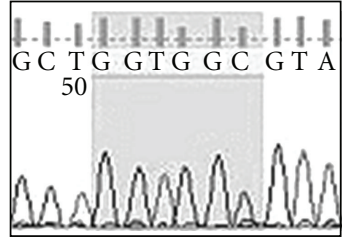

(b)

FIGURE 1: The electropherograms of the SNaPshot analysis (a) and the DNA sequencing (b) of the same tumor tissue sample. The mutation of codon 12 position 2 guanine to adenine transition is definitely visible in the SNaPshot analysis. In the sequencing analysis, the adenine-peak is hardly distinguishable from the background.

2.5. Statistical Analysis. The sensitivity and the specificity of SNaPshot analysis in KRAS mutation screening in comparison to common DNA sequencing was tested. The DNA sequencing was assumed as standard method with a specificity and sensitivity of 100\%.

\section{Results}

In $45(45 \%)$ out of 100 analysed tissue samples, a KRAS mutation in codon 12 or 13 were detected. 40 (88.9\%) of these mutated KRAS genes were discovered by DNA sequencing and SNaPshot, a further five mutations (11.1\%) were only detectable by SNaPshot. This results in a sensitivity of $100 \%$ with a confidence interval of $95 \%$ [91.2\% to $100 \%$ ] and a specificity of $91.7 \%$ with a confidence interval of $95 \%$ [81.6\% to $97.2 \%$, respectively.

Figure 1 shows an example of a mutation detection by $\mathrm{SNaPshot} \mathrm{but} \mathrm{not} \mathrm{by} \mathrm{sequencing.} \mathrm{The} \mathrm{analysed} \mathrm{cancer}$ specimens were dissected in NSCLC, colorectal carcinoma, and pancreatic carcinoma. For NSCLC, we detected in 15/41 (36.6\%) KRAS-mutated cancer tissue samples, in 6/20 (30\%) KRAS mutations in colorectal specimens, in 14/19 (73.7\%) pancreatic tumor samples, and in 9/20 (45\%) colorectal tumor samples from the interlaboratory comparison.

The type of mutations in KRAS exon 2 codons 12 and 13 can be grouped in transitions and transversions. Transition mutations of KRAS exon 2 codons 12 and 13 occur in the majority $(71.4 \%)$ of pancreatic carcinoma. NSCLC KRAS mutations mainly occur as transversions (78.6\%) and KRAS mutations of colorectal carcinomas are evenly distributed as transition and transversion. In the NSCLC samples, G37T, G38A, G35T G34T, and G35A occur. The colorectal specimens show G34A, G38C, G35A, and G34T mutations. The pancreatic carcinomas show three different types of KRAS mutations: G35A, G34C, and G35T. The kind of KRAS mutations found in the tissue samples are explicitly listed in Figure 2 with the percentage of the total mutation number.

\section{Discussion}

The given percentage of KRAS mutation in NSCLC is 21$43 \%[4,14]$ and $33 \%$ [15] for colorectal cancer and for pancreatic carcinomas $75-82.4 \%[15,16]$. KRAS mutation frequency in our examinations showed $36.6 \%$ for NSCLC and $30 \%$ for colorectal cancer. $73.7 \%$ of mutated KRAS genes in pancreatic carcinoma is in accordance with previously described data [15].

With regard to the present prerequisite of KRAS mutation screening before a therapy with Panitumumab and Cetuximab, KRAS is a powerful molecular marker in cancer diagnostics $[1,5]$. The molecular cancer diagnostic is on the way to afford a more individualised therapy for these patients. This aim makes a molecular screening of the tumor necessary, which reveals the cancer characteristics correlated to the response to a chemotherapy.

Several studies indicate greater benefit from a Cetuximab therapy for EGFR expressing metastatic colorectal cancer patients with KRAS wild type [5-7]. Cetuximab in the firstline treatment combined with Oxaliplatin, Leucovorin, and Fluorouracil increases the overall response rate in patients with metastatic colorectal cancer [17].

Next to the important prediction of drug response for metastatic colorectal carcinomas, KRAS mutations may also be decisive for drug decision in NSCLC in regard to Erlotinib or Gefitinib, because these kinase inhibitors seem to be ineffective in carcinomas with KRAS mutation $[12,16,17]$. Eberhard et al. [14] showed a shorter median time to progression and survival in patients treated by Erlotinib and Cisplatin in case of mutated KRAS compared to patients receiving Cisplatin alone. KRAS does not seem to be a predictive factor [15] in a study with Gefitinib and chemotherapy for advanced colorectal cancer.

Particularly with regard to the expanding field of KRAS mutation status detection, it is necessary to determine the allele status in a reliable way. Therefore, knowledge of the KRAS gene mutation status is essential before the decision is made to treat a cancer patient using EGFR-targeted Panitumumab and Cetuximab therapy. Also considering the enormous costs and side effects of the therapy, the KRAS gene mutation analysis is necessary.

KRAS mutation can be screened in several ways: DNA sequencing, commercialised test systems by DNA-DNA hybridisation and SNaPshot analysis [8]. In this paper, we compare the common sequencing analysis and the SNaPshot, which is regarded to be more reliable [8]. Our results do reveal a better mutation detection, shown by the statistical analysis. The sensitivity reached $100 \%$ and the specificity $91.7 \%$, implying a high probability of accuracy of the SNaPshot. 


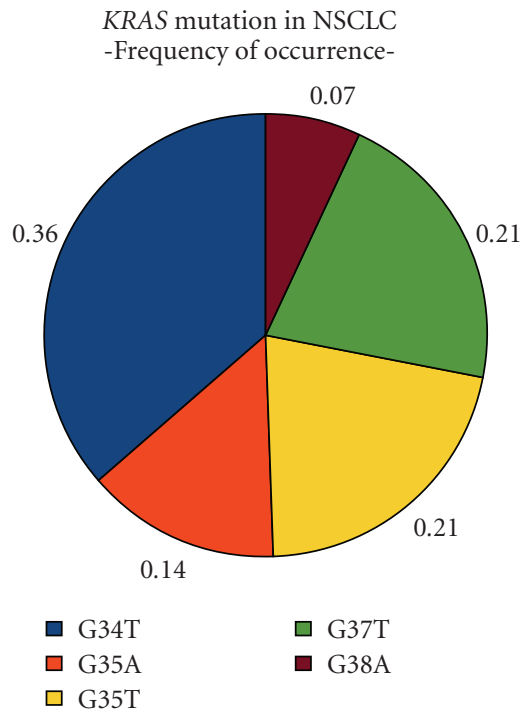

(a)

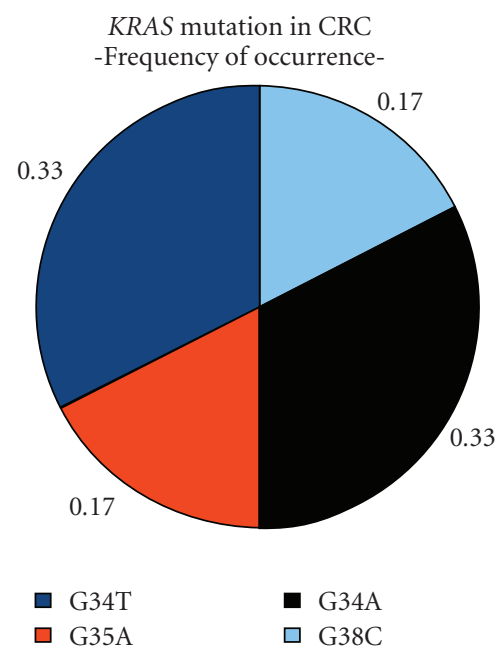

(b)
KRAS mutation in pancreatic carcinoma -Frequency of occurrence-

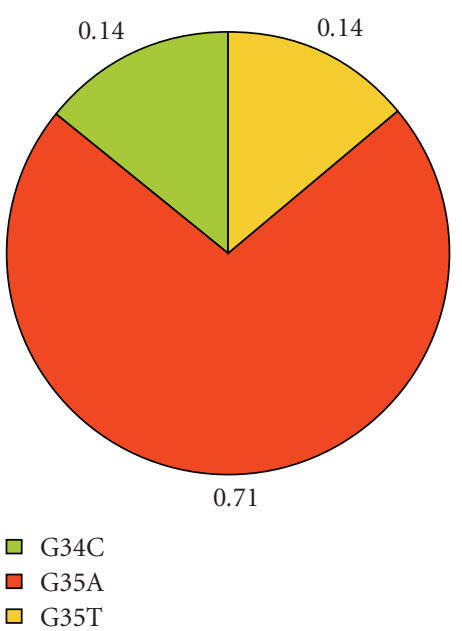

(c)

Figure 2: Delaminating of KRAS genotype of different tissue specimen. This picture shows the percentage of mutation types found in the particular kind of cancer specimens. The legend under the picture explains the colours used for the different genotypes.

To ensure a positive mutation result and avoid false positive results, it is also recommended to run, next to a nucleic acid-free control sample ("water-control"), a KRAS wildtype control, with the known wildtype KRAS sequence.

The SNaPshot analysis shows a few advantages in addition to the enhanced reliability. It is less expensive and more rapid than the sequencing analysis (SNaPshot $11 €$ versus $19 €$ for DNA sequencing) and could be analysed without additional software, except the Genemapper program (Applied Biosystems, Forster City, CA, USA). Considering these qualities, the SNaPshot analysis could replace DNA sequencing in KRAS mutation screening.

The Therascreen: K-RAS Mutation Kit from Qiagen [12] uses a combination of ARMS-technology and Scorpionsprimers, so it is possible to determine mutations of only $1 \%$ mutated allel in the background of wildtype genotype. But the kit could detect only 7 possible mutations types of the KRAS gene, but a whole of 12 mutations can occur in codons 12 and 13 . In our analysis, we detected 2 mutations types, one in a colorectal carcinoma specimen, which would be detected as false negative with the therascreen kit.

The KRAS mutations can be subdivided in transversions and transitions. Both events lead to the exchange of the amino acid glycine to another amino acid in the protein. A transition is a replacement of one purine base to the other or one pyrimidine base to another, and a transversion is a replacement from a purine base to a pyrimidine base or vice versa. The occurrence of transition and transversion is not evenly distributed in these three different kinds of tumor tissue. Transversions appear more frequently in NSCLC and transitions more frequently in pancreatic carcinoma. Both mutation types occur evenly in colorectal carcinoma. An unequal frequency of occurrence of mutation types suggests different conditions in these three kinds of tumor. For a transition, just the addition or removal of an amino group is necessary. For a transversion, the base has to be removed and the new base has to be added. Riely et al. showed transition of the KRAS gene in lung cancer occurring more frequent in never smokers than in former or current smokers. This could imply that transversion mutations are associated with smoking [18].

For protocol of the KRAS detection only by SNaPshot, is it feasible to shorten the purification step of the PCR and use only a SAP/EXO degradation of interfering nucleic acids. Depending on the size of the PCR product, the SNaPshot is expandable to newly identified SNPs, simply by adding a suitable primer and PCR product to the reaction. This is a less expensive method to analyse SNPs in other genomic regions by just adding primer for the PCR and the SNaPshot reaction. However, the data cannot be reanalysed for unconsidered sequences. The SNaPshot has to be repeated with additional primers.

Tiny proportions of mutated KRAS alleles can easily be screened by the above-mentioned methods. In event of KRAS mutation, therapy with monoclonal antibodies Cetuximab and Panitumumab should be withdrawn $[5,11]$. Tiny allele amounts of mutated KRAS suggest a majority of wild type cells concerning the KRAS gene in the tumor. The lack of quantification of KRAS mutation alleles may deprive patients of a treatment, which could be targeted on tumor cells with intact KRAS gene. Therefore, KRAS mutation detection with SNaPshot could have a more enhanced role in response prediction of therapy depending on which response can be expected due to the amount of mutated KRAS allele in the tumor. 


\section{Conflict of Interests}

The authors indicated no potential conflicts of interests.

\section{References}

[1] R. G. Amado, M. Wolf, M. Peeters et al., "Wild-type KRAS is required for panitumumab efficacy in patients with metastatic colorectal cancer," Journal of Clinical Oncology, vol. 26, no. 10, pp. 1626-1634, 2008.

[2] M. Peeters, J. Balfourf, and D. Arnold, "Review article: panitumumab-a fully human anti-EGFR monoclonal antibody for treatment of metastatic colorectal cancer," Alimentary Pharmacology and Therapeutics, vol. 28, no. 3, pp. 269-281, 2008.

[3] R. M. Giusti, K. A. Shastri, M. H. Cohen, P. Keegan, and R. Pazdur, "FDA drug approval summary: panitumumab (Vectibix)," Oncologist, vol. 12, no. 5, pp. 577-583, 2007.

[4] http://www.emea.europa.eu/pdfs/human/opinion/ 40511307en.pdf.

[5] A. Lièvre, J. B. Bachet, D. Le Corre et al., "KRAS mutation status is predictive of response to cetuximab therapy in colorectal cancer," Cancer Research, vol. 66, no. 8, pp. 39923995, 2006.

[6] A. Lièvre, J. B. Bachet, V. Boige et al., "KRAS mutations as an independent prognostic factor in patients with advanced colorectal cancer treated with cetuximab," Journal of Clinical Oncology, vol. 26, no. 3, pp. 374-379, 2008.

[7] W. De Roock, H. Piessevaux, J. De Schutter et al., "KRAS wild-type state predicts survival and is associated to early radiological response in metastatic colorectal cancer treated with cetuximab," Annals of Oncology, vol. 19, no. 3, pp. 508$515,2008$.

[8] F. Di Fiore, F. Blanchard, F. Charbonnier et al., "Clinical relevance of KRAS mutation detection in metastatic colorectal cancer treated by Cetuximab plus chemotherapy," British Journal of Cancer, vol. 96, no. 8, pp. 1166-1169, 2007.

[9] J. L. Bos, "ras oncogenes in human cancer: a review," Cancer Research, vol. 49, no. 17, pp. 4682-4689, 1989.

[10] S. Schubbert, K. Shannon, and G. Bollag, "Hyperactive Ras in developmental disorders and cancer," Nature Reviews Cancer, vol. 7, no. 4, pp. 295-308, 2007.

[11] S. Ogino, T. Kawasaki, M. Brahmandam et al., "Sensitive sequencing method for KRAS mutation detection by pyrosequencing," Journal of Molecular Diagnostics, vol. 7, no. 3, pp. 413-421, 2005.

[12] http://www.dxsdiagnostics.com/Site/PDF/CMP/TheraScreen KRAS/IFU-KRAS-TheraScreen-ZA.pdf.

[13] W. Weichert, C. Schewe, A. Lehmann et al., "KRAS genotyping of paraffin-embedded colorectal cancer tissue in routine diagnostics: comparison of methods and impact of histology," Journal of Molecular Diagnostics, vol. 12, no. 1, pp. 35-42, 2010.

[14] D. A. Eberhard, B. E. Johnson, L. C. Amler et al., "Mutations in the epidermal growth factor receptor and in KRAS are predictive and prognostic indicators in patients with nonsmall-cell lung cancer treated with chemotherapy alone and in combination with erlotinib," Journal of Clinical Oncology, vol. 23, no. 25, pp. 5900-5909, 2005.

[15] S. Ogino, J. A. Meyehardt, M. Cantor et al., "Molecular alterations in tumors and response to combination chemotherapy with gefitinib for advanced colorectal cancer," Clinical Cancer Research, vol. 11, no. 18, pp. 6650-6656, 2005.
[16] J. Ugorčáková, T. Hlavatý, P. Babál et al., "Assay design and optimization of mutant-enriched PCR based method for detection of K-ras gene mutations in pancreatic carcinoma," Neoplasma, vol. 53, no. 5, pp. 363-371, 2006.

[17] C. Bokemeyer, I. Bondarenko, A. Makhson et al., "Fluorouracil, leucovorin, and oxaliplatin with and without cetuximab in the first-line treatment of metastatic colorectal cancer," Journal of Clinical Oncology, vol. 27, no. 5, pp. 663$671,2009$.

[18] G. J. Riely, M. G. Kris, D. Rosenbaum et al., "Frequency and distinctive spectrum of KRAS mutations in never smokers with lung adenocarcinoma," Clinical Cancer Research, vol. 14, no. 18, pp. 5731-5734, 2008. 


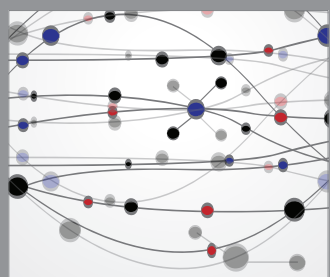

The Scientific World Journal
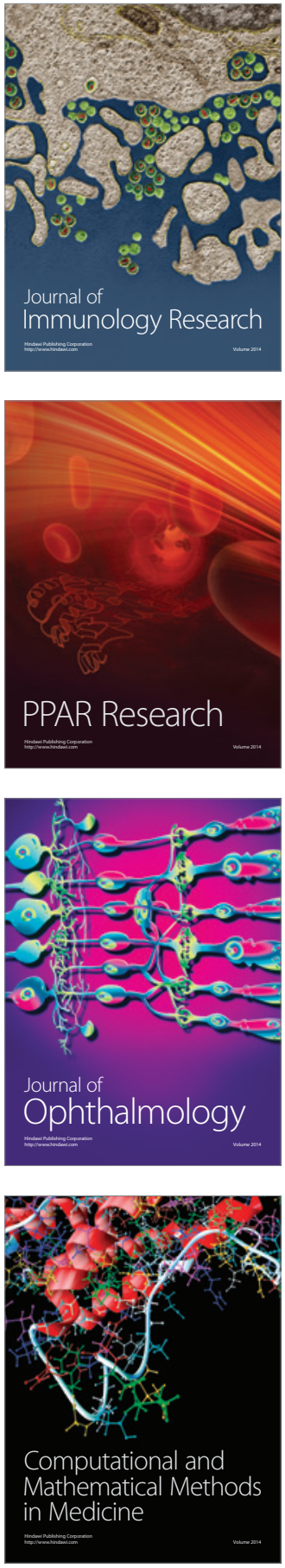

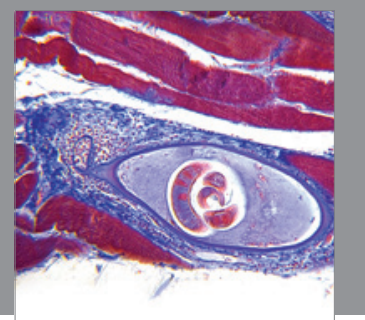

Gastroenterology

Research and Practice
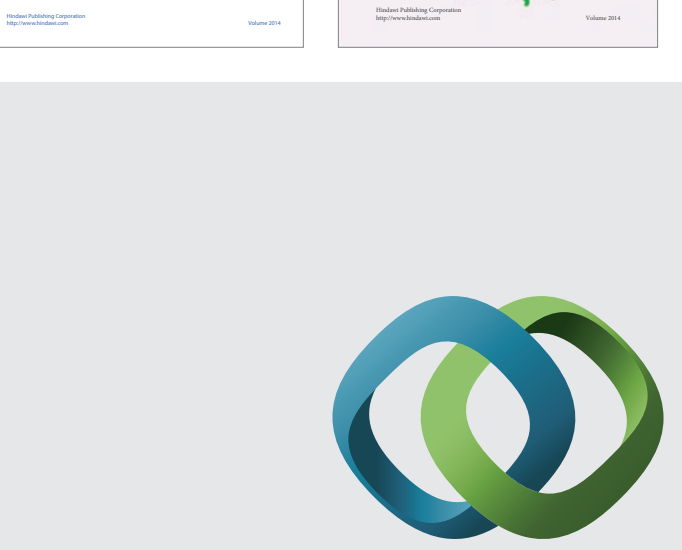

\section{Hindawi}

Submit your manuscripts at

http://www.hindawi.com
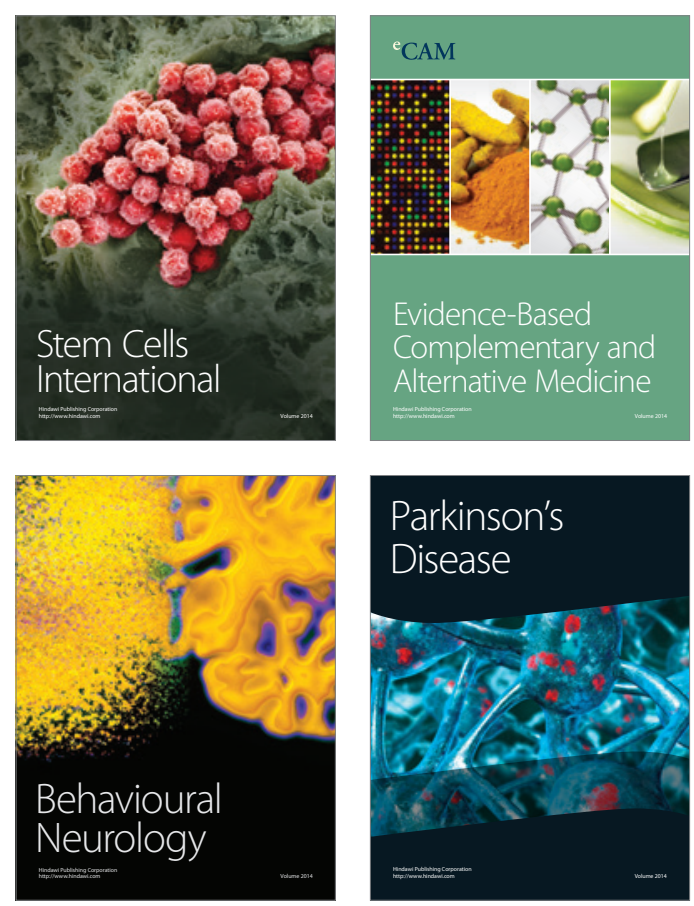

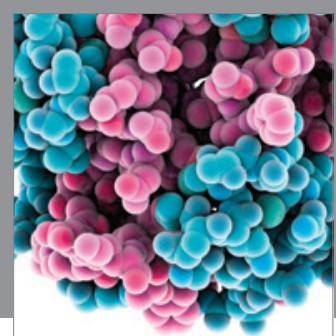

Journal of
Diabetes Research

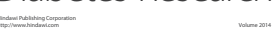

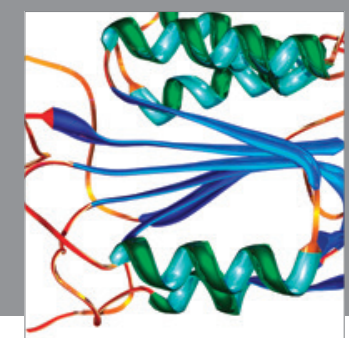

Disease Markers
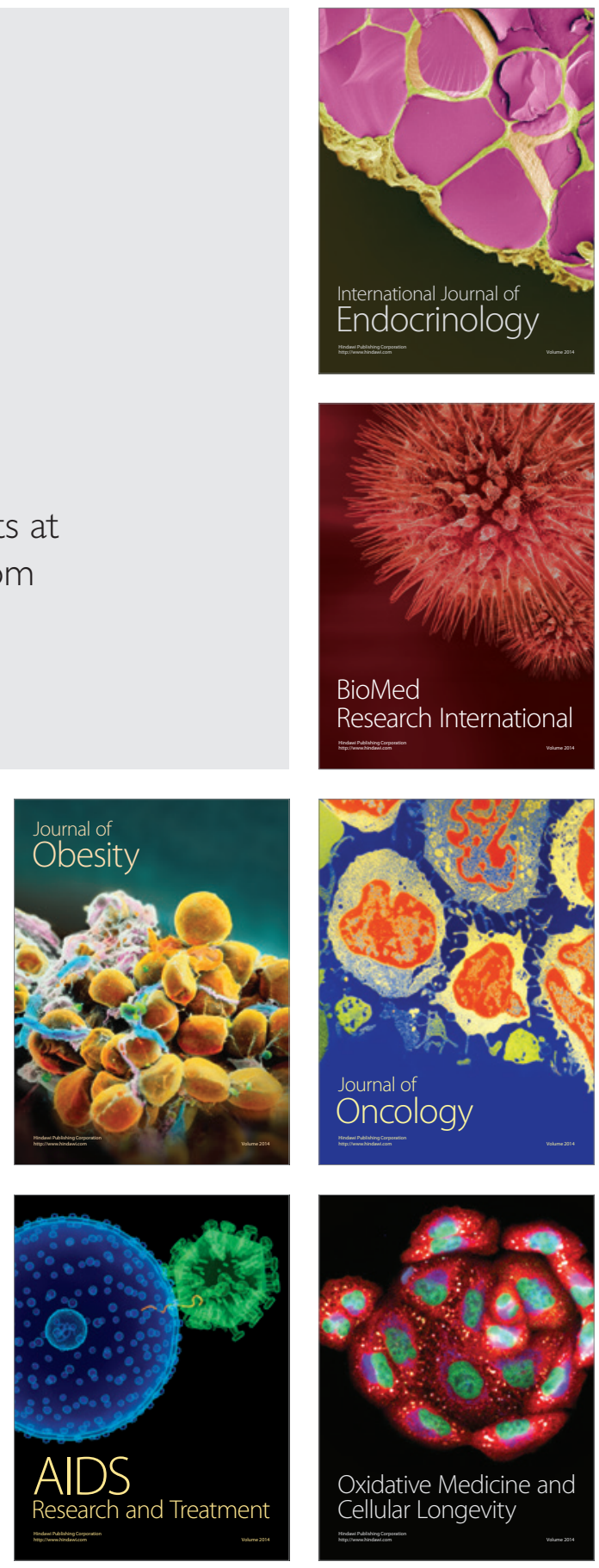